\title{
An Atypical Case of Pityriasis Rosea Gigantea after Influenza Vaccination
}

\author{
Dimitrios Papakostas ${ }^{a} \quad$ Panagiotis G. Stavropoulos ${ }^{a} \quad$ Dafni Papafragkakia \\ Ekaterini Grigoraki $^{b} \quad$ Georgia Avgerinou $^{a} \quad$ Christina Antoniou $^{a}$ \\ ${ }^{a}$ Department of Dermatology, A. Syggros Hospital, and ${ }^{b}$ Department of Histopathology, \\ University of Athens, Athens, Greece
}

\section{Key Words}

Pityriasis rosea - Vaccination - Human herpes virus- 6 - Human herpes virus-7 ·

Suberythrodermia

\section{Abstract}

Pityriasis rosea is a common erythematosquamous eruption, typically presenting along the cleavage lines of the skin. A wide spectrum of atypical manifestations may challenge even the most experienced physician. Here we report a rare case of a suberythrodermic pityriasis rosea with gigantic plaques after an influenza vaccination, and we discuss the possible triggers of atypical manifestations of such a common dermatological disease in the setting of an altered immunity.

(c) 2014 S. Karger AG, Basel

\section{Introduction}

Pityriasis rosea is a common, self-limited erythematosquamous eruption that favors the trunk and proximal extremities of young adolescents. The characteristic 'herald' lesion precedes the 'blossoming' that takes place, and, after a couple of days, results in the typical 'christmas tree' pattern of the pityriasis rosea plaques along the cleavage lines of the skin. In classical cases of the disease, the lesions appear as erythematous sharply demarcated plaques, covered by fine 'collarette'-like scales that heal spontaneously after an average of 6 - 8 weeks. Variations in size, morphology of the plaques, location and distribution or even prolonged disease duration with relapses, result in a wide clinical spectrum of disease manifestations and may challenge even the most experienced dermatologists [1, 2].

Higher prevalence in healthy young adults, self-limited course with rare relapses, prodromal symptoms, case clustering and seasonal variations consisting of small 'outbreaks' in

Dr. Dimitrios Papakostas

Department of Dermatology

A. Syggros Hospital, University of Athens

5 I. Dragoumi Str., GR-16121 Athens (Greece)

E-Mail dimitrios.s.papakostas@gmail.com 
spring and fall suggest an infectious etiology [3]. Although a number of viral agents has been investigated so far, the etiologic correlation of the disease with a specific virus has not yet been established. However, recent findings support the viral hypothesis and suggest that the reactivation of human herpes virus-6 (HHV-6) and human herpes virus-7 (HHV-7) may contribute to the development of acute skin lesions [4].

\section{Case Report}

A 71-year-old male presented with the complaint of an itchy rash over his upper trunk, his upper and lower extremities and the abdomen for the last 2 weeks. The rash appeared 1 month after his seasonal influenza immunization, initially on the trunk, and it subsequently spread to the extremities. It worsened after the application of emollients and after warm showers. At examination, the patient was negative for fever, weight loss, photosensitivity, shortness of breath, abdominal pain or arthralgias. His medical history included paroxysmal atrial fibrillation, hypertension and dyslipidemia. He denied taking any new medication or illicit drugs, and his social history was negative for smoking or alcohol abuse.

On clinical examination, the patient appeared anxious, but in general good health. His rash consisted of confluent large salmon-colored plaques with a mild induration and a fine scale over his trunk and shoulders, discrete small circular or oval lesions along the cleavage lines, on the upper trunk and on the shoulder erythrodermia were visible. He also showed plaques over his forearms and tibiae (fig. 1). No cervical, axillary or inguinal lymphadenopathy was noted. His palms and soles were free of lesions and there was no mucosal involvement.

The laboratory work-up, including complete blood count and basic metabolic profile, was within normal limits. The patient had a biopsy done on his upper back which showed mild hyperkeratosis and focal epidermal parakeratosis, mild spongiosis, extensive intracellular edema and focal lymphocytic exocytosis, moderate inflammatory infiltrate from the lymphocytes and histiocytes in his upper dermis, and a few polymorphonuclear cells perivascularly. Immunohistochemistry was positive for an intracellular accumulation of both HHV-6 and HHV-7 (fig. 2). In situ hybridization allowed the localization of multiple copies of HHV-6 and HHV-7 in the cytoplasm of many epidermal cells.

The diagnosis of eczematous pityriasis rosea gigantea was made based on the patient's history, and clinical and histopathological evidence. The patient received oral antihistamines, topical corticosteroids and 1 intramuscular injection of betamethasone. His rash gradually improved and eventually cleared completely over a period of 3 months.

\section{Discussion}

The sudden appearance of confluent, large erythematosquamous plaques developing to a suberythrodermia made the exclusion of a cutaneous T-cell lymphoma like mycosis fungicides necessary $[5,6]$. Other causes of suberythrodermia like psoriasis vulgaris, atopic eczema and drug-associated rushes were considered, but there was no evidence for that by looking at the patient's history.

Sporadic cases of the so-called pityriasis rosea gigantea or giant pityriasis rosea have already been reported in the literature [7]. Further, larger plaques can be seen in another very rare variant of the pityriasis rosea, the inverse pityriasis rosea of Vidal with multiple 
Papakostas et al.: An Atypical Case of Pityriasis Rosea Gigantea after Influenza Vaccination

large oval or round annular eruptions, ranging from 3-6 cm in diameter, typically present in the axillary region or the groins and sparing the trunk and the extremities [8].

The diagnosis of a giant pityriasis rosea was supported by the self-limited course of the disease, the compatible histology without any signs for a malignancy or fungal infection, and positive immunohistochemistry and in situ hybridization for HHV-6 and HHV-7. The viral etiology of pityriasis rosea has been extensively discussed. Light and electron microscopy observation of intranuclear and intracytoplasmic virus-like particles and the detection of cytolytic degeneration of keratinocytes suggest viral implication [9]. The predominance of Tcell-mediated immunity suggests a pathophysiologic mechanism involving the interaction of T-helper cells, Langerhans cells, and inflammatory dendritic epidermal cells, in a setting triggered by viruses [10]. Recently, research has focused on HHV-6 and HHV-7. In most cases, after primary infection in early childhood, these viruses establish a latent infection in a specific set of cells of their host and are capable of reactivation in conditions of altered immunity [11]. They are widely encountered in the general adult population (80-90\%). HHV-6 and HHV-7 DNA has been found in the serum, HHV- 6 and HHV-7 antigens have been detected in skin lesions by immunohistochemistry, and the presence of viral mRNA has been documented by in situ hybridization. The above indicate a viral replication, suggesting that, in the acute stages, an active infection may be present as a result of virus reactivation, similar to herpes simplex or varicella zoster virus $[4,9]$. Furthermore, there is evidence that HHV-6 and HHV-7 may interact with each other [12].The interaction of the 2 viruses might explain the small number of recurrences and the multitude of atypical presentations.

The mechanisms that trigger the reactivation of the 2 viruses remain obscure. However, pityriasis rosea-like rashes after vaccinations have been reported in literature. Recently, Chen et al. [13] reported a clinicopathologically typical case of pityriasis rosea that developed after H1N1 vaccination. The herald patch developed 5 days after vaccination and was followed several days later by the onset of many itchy scaly lesions on the trunk and the proximal extremities. As far as pathogenetic mechanisms behind the triggering of pityriasis rosea after vaccination are considered, we could support that vaccine-induced immune stimulation may trigger a reactivation of latent viral agents such as HHV-6 and HHV-7 or that molecular mimicry with a viral epitope could result in a T-cell-mediated skin reaction. Nonetheless, the apparently multifactorial pathogenesis of pityriasis rosea, which could be further affected by concomitant medical treatments and the immune status of the patient in case of co-infections or immunosuppression, could easily explain the wide spectrum of atypical presentations of this common dermatological disease.

\section{Conclusion}

We reported a very rare case of pityriasis rosea with multiple gigantic herald plaques after influenza vaccination. In a state of altered immunity, reactivation of HHV-6 and/or HHV-7 could result through a T-cell-mediated skin-oriented reaction in an atypical presentation of a self-limited suberythrodermic gigantic pityriasis rosea.

\section{References}

1 Zawar V, Chuh A: Follicular pityriasis rosea. A case report and a new classification of clinical variants of the disease. J Dermatol Case Rep 2012;6:36-39.

2 Chuh A, Zawar V, Lee A: Atypical presentations of pityriasis rosea: case presentations. J Eur Acad Dermatol Venereol 2005;19:120-126. 
Papakostas et al.: An Atypical Case of Pityriasis Rosea Gigantea after Influenza Vaccination

-3 Canpolat Kirac B, Adisen E, Bozdayi G, et al: The role of human herpesvirus 6, human herpesvirus 7, EpsteinBarr virus and cytomegalovirus in the aetiology of pityriasis rosea. J Eur Acad Dermatol Venereol 2009;23:16-21.

4 Rebora A, Drago F, Broccolo F: Pityriasis rosea and herpesviruses: facts and controversies. Clin Dermatol 2010;28:497-501.

5 Browning JC: An update on pityriasis rosea and other similar childhood exanthems. Curr Opin Pediatr 2009;21:481-485.

6 Wollenberg A, Eames T: Skin diseases following a Christmas tree pattern. Clin Dermatol 2011;29:189-194.

7 Zawar V: Giant pityriasis rosea. Indian J Dermatol 2010;55:192.

8 Zawar V, Godse K: Groin eruptions: pityriasis rosea of vidal. Int J Dermatol 2011;50:195-197.

-9 Drago F, Broccolo F, Rebora A: Pityriasis rosea: an update with a critical appraisal of its possible herpesviral etiology. J Amer Acad Dermatol 2009;61:303-318.

10 Neoh CY, Tan AWH, Mohamed K, et al: Characterization of the inflammatory cell infiltrate in herald patches and fully developed eruptions of pityriasis rosea. Clin Exp Dermatol 2010;35:300-304.

11 Drago F, Rebora A: The new herpesviruses: emerging pathogens of dermatological interest. Arch Dermatol 1999;113:71-75.

12 Katsafanas GC, Schirmer EC, Wyatt LS, et al: In vitro activation of human herpesviruses 6 and 7 from latency. Proc Natl Acad Sci USA 1996;93:9788-9792.

13 Chen JF, Chiang CP, Chen YF, Wang WM: Pityriasis rosea following influenza (H1N1) vaccination. J Chin Med Assoc 2011;74:280-282.

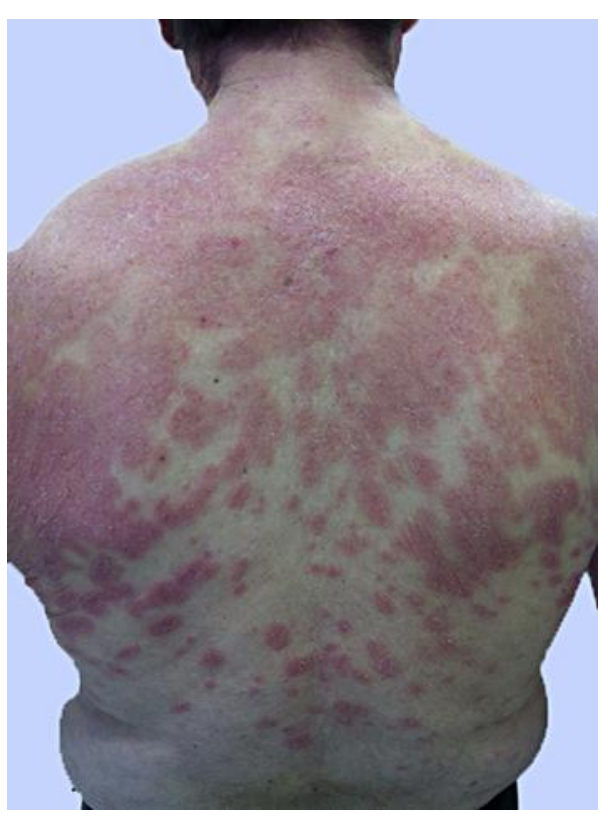

Fig. 1. Erythrodermia on the shoulders; confluent large salmon-colored plaques with mild induration and fine scales on the upper trunk, and discrete small circular or oval lesions along the cleavage lines on the lower trunk of the patient. 
Papakostas et al.: An Atypical Case of Pityriasis Rosea Gigantea after Influenza

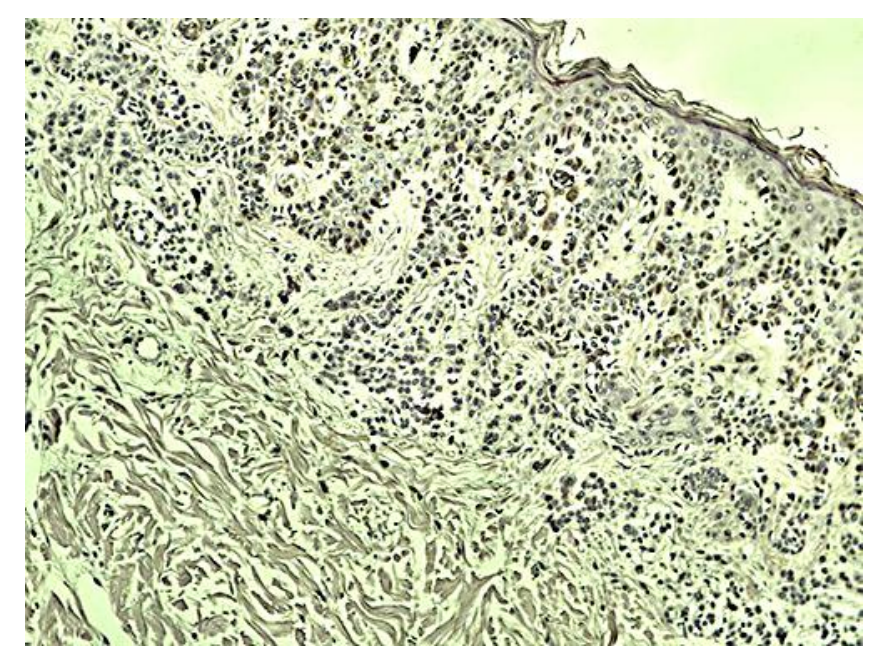

Fig. 2. Mild hyperkeratosis and focal epidermal parakeratosis, mild spongiosis, extensive intracellular edema and focal lymphocytic exocytosis, moderate inflammatory infiltrate from the lymphocytes and histiocytes in his upper dermis and few polymorphonuclear cells perivascularly. Immunohistochemistry showed an intracellular accumulation of HHV-6 (brown stain). 